\title{
Simplifying Mobile Phone Food Diaries
}

\section{Design and Evaluation of a Food Index-Based Nutrition Diary}

\author{
Adrienne H. Andrew, Gaetano Borriello, James Fogarty \\ Computer Science \& Engineering \\ DUB Group, University of Washington \\ aha@aro.com, gaetano@cs.washington.edu, jfogarty@cs.washington.edu
}

\begin{abstract}
We describe the design and evaluation of POND, a Pattern-Oriented Nutrition Diary. POND is a mobile-phone food diary designed using a theory-driven approach to address a common challenge users report when using food diaries on mobile phones: the amount of effort required to create food entries in relation to the perceived self-benefit of self-monitoring food intake. The design allows users to create food entries either via a traditional database lookup or a streamlined ' +1 ' approach. 24 people used POND to create predefined food entries. We found people preferred different approaches to creating entries, which reflected their self-reported nutrition concerns. This supports an argument for rethinking traditional approaches to designing food diaries.
\end{abstract}

Keywords—mobile health and wellness; theory-driven design; nutrition; self-monitoring.

\section{INTRODUCTION}

Globally, the incidence of lifestyle diseases such as obesity, diabetes, and cardiovascular disease are increasing. One-third of adults were overweight or obese in 2008 [9]. Additionally, it is estimated at least $50 \%$ of cancers are preventable by encouraging healthy behaviors and discouraging unhealthy practices [2]. Researchers believe that a primary cause of obesity is an imbalance of energy intake and expenditure: people eat too many calories while not burning enough. While obesity is a risk factor for cardiovascular disease, diabetes, and cancer, it is believed these diseases are also impacted by the kinds of food people eat. In addition to balancing caloric intake, the American Heart Association recommendations for preventing cardiovascular disease include consuming a diet rich in fruits and vegetables, choosing whole grain-high fiber foods, limiting saturated fat intake, and reducing consumption of added sugars and alcohol [10]. Similar recommendations are made for preventing diabetes [19].

Persuasive technology aims to use technology to persuade or nudge people to change their behavior [7]. Ubiquitous mobile devices, such as smartphones, have been considered ideal platforms for administering behavior change programs, especially around eating behaviors. In September 2012, the Pew Internet and American Life project reported that $45 \%$ of American adults own smartphones [15]. Because smartphones are personal devices usually kept with or near people, they enable kairos: providing relevant information and decision support at the time of need. In other words, these devices allow people to self-monitor what they eat by providing a means to capture what has already been eaten. Devices can also provide timely support for looking up caloric values for prospective foods, allowing people to make an informed

Adrienne Andrew is now affiliated with ARO, Inc. decision about what to eat, before they eat it. However, formal research and informal feedback indicates people have difficulties adhering to the use of mobile-phone food diaries for extended periods of time. We want to examine new techniques that could enable longer-term use of mobile-phone food diaries.

The contributions of this work are twofold. First, we present the design of a food diary that does not depend on a food database. Second, we report on an initial in-lab usability study.

\section{RELATED WORK}

Related work in self-monitoring of dietary intake falls in three areas: a focus on a specific disease population; the use of photography to capture and analyze food intake; and incorporating strategies to summarize dietary intake.

\section{A. Food Tracking for Special Populations}

Siek et al. [17,18] explored PDA-based self-monitoring of dietary intake by individuals with chronic kidney disease. They examined when people made entries, what challenges they faced, and the use of barcode scanners and voice input to improve food entry. Mamykina et al. [11,12] explored supporting diabetes patients in managing their dietary intake. Newly diagnosed diabetics usually go through a period of changing their dietary behaviors. MAHI is a mobile phone application that supports users in capturing and documenting eating episodes throughout the day. The records can be reviewed later, encouraging reflection on behavior and choices. This can be characterized as a quick-capture with a strong emphasis on post-hoc analysis. The user populations in the Siek and Mamykina work have well-defined constraints on dietary intake. People who need to change nutrition behaviors to treat a disease may be more motivated to use technology to support self-monitoring than individuals focused on preventing disease.

\section{B. Tracking with Photography}

Another approach to simplifying the food logging procedure is to use photos. This approach consists of two phases: capturing food images and processing the images to identify nutritional intake. Food images can be captured actively by the user with a mobile phone camera $[1,5,20]$, or passively with a lifelogging camera or mobile phone app [16]. Identifying foods, amounts, and nutritional values from the food images can be done either by crowd sourcing [14] or automated computer vision approaches [6]. The work presented in this paper focuses on the amount of detail captured in a food diary. Our work could inform the amount of detail captured or displayed in photography-based approaches. Additionally, while most photography approaches have the goal of minimizing user 


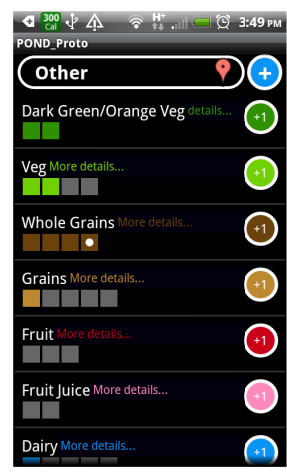

(a)

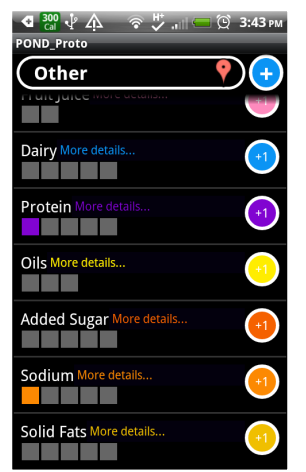

(b)

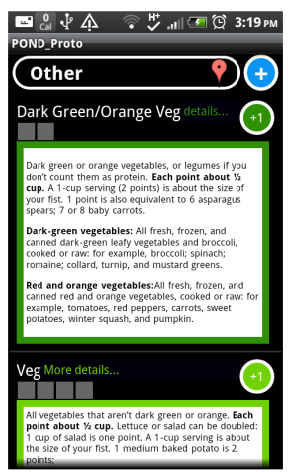

(c)

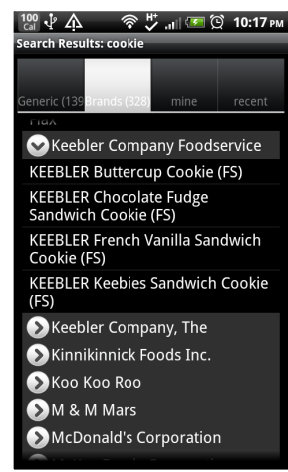

(d)

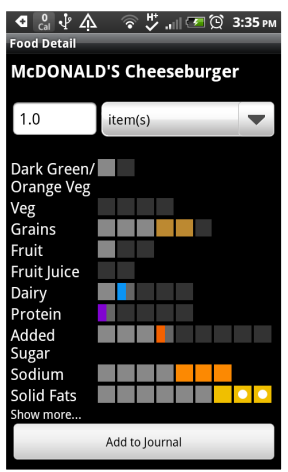

(e)

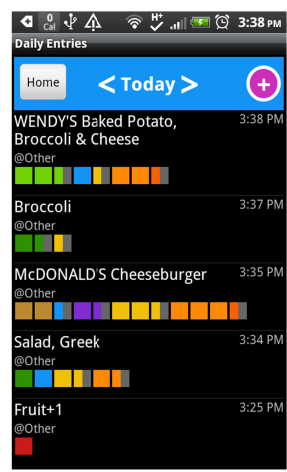

(f)

Figure 1. An overview of the POND interface: $(a, b)$ The main screen includes presents each component, the recommended portions, and the consumed portions.

(c) A details screen provides a description of a component and example serving sizes. (d, e) A database supports looking up the component counts of foods.

(f) A review screen presents a list of foods that have been entered for the current day and allows editing of entries.

involvement in the capture process, it is unclear how behavior change depends on the user being actively involved in the self-monitoring process.

\section{Tracking Summarized Measures}

In contrast to tracking detailed caloric intake and expenditure, the Wellness Diary [13] allowed users to monitor many different health and wellness indicators. These include quality of food intake, amount of physical activity, sleep, stress levels, and amount of time spent at work. In regards to the self-monitoring of dietary intake, eating episodes were captured by triage: a meal could be rated as "healthy", "not healthy", or "unknown". This study suggests even cursory attention to monitoring food intake can have impact on eating behaviors. This is consistent with work by Burke et al. [4] who studied participants in a behavioral weight-loss program and found varying levels of engagement with the self-monitoring process. Some participants were highly engaged, while others nominally followed the procedure or were inconsistent. It is unclear whether this is due to internal or external factors. However, a self-monitoring tool that allows for varying levels of engagement could support a wider range of users.

Andrew et al. [3] compared user performance and preference for three different food diaries: a traditional database-lookup diary; a diary tracking only food groups; and a diary tracking food groups and some nutrients. They found that in a lab study, users made the fewest errors with the traditional food diary, but took the most time. The food group diary took the least time, with an error rate similar to the traditional diary. However, users felt the food group diary was over-simplified. The reduced time and errors were not worth the loss of detail. Users preferred either the traditional approach or the food groups plus nutrients approach.

\section{BACKGROUND}

The Healthy Eating Index (HEI) [8] is a food index based on the USDA 2005 Dietary Guidelines. The HEI is a score-based system, with a diet that closely reflects the Dietary Guidelines earning a score of 100 points. Twelve components contribute to the overall score: whole grains; all grains; dark green and orange vegetables; all other vegetables; whole fruit; all other fruit products; meat, beans and eggs; dairy; oils; sodium; saturated fat; and discretionary calories from solid fats, added sugars and alcohol. Some components are attainment components, where the score goes up as more of that food is eaten, reflecting that most
Americans do not eat enough of these foods (such as whole grains or vegetables). Other components are to be moderated, causing the score to decrease as more is consumed, reflecting that most Americans eat too much of these components (such as discretionary calories). The attainment components for HEI contain primarily food groups. All of the moderation components are considered nutrients. Calculation for each point depends on an individual's daily recommended caloric intake.

\section{THE DESIGN OF POND}

We present the design of POND, a Pattern-Oriented Nutrition Diary. POND is an Android app that explores the value of reducing the amount of time and effort to capture an eating episode while also reducing the amount of detail that is captured. The primary goal informing the design of POND was to minimize the amount of time it took to make an entry. Preference was given to design decisions that minimized initial food entry creation.

POND's design reflects the HEI. It allows users to focus on monitoring components of food they eat. Users enter food by counting the contribution to each of 13 components, rather than a more traditional approach of looking up a food in a database. The POND components differ from the HEI in that the HEI discretionary calories component was split into separate solid fats, added sugar, and alcohol components. This was based on early user feedback which indicated users found it easier to track calories for each of these items separately. Since there is a close relationship between solid fats and saturated fat, these two components were combined into one solid fat component. Users can customize the interface to present and track all components, or they can hide components which they are less interested in tracking.

Figure 1 shows the initial screen the user sees when they launch POND. This was designed to prioritize quick entry and quick analysis of the current progress toward goals for the day. Each row represents a recommendation based on the HEI. Dark gray blocks indicate the daily goal for that component. A colored block indicates how many portions of that component have been consumed. A colored block with a white dot indicates the user has consumed more than the goal number of servings. The use of a white dot in the block was chosen to provide neutral feedback about the number of servings consumed. Users touch the +1 buttons on the right side of the screen to quickly indicate a portion eaten, or long-press the +1 buttons to indicate a half-portion eaten. 
Colored links next to the component name expand to show more detailed information about that component, including how much to count as one block (see Figure 1c). This information was adapted from the USDA 2010 Dietary Guidelines. Blocks represented familiar serving sizes represented by real-world objects (e.g. "a pancake the size of a DVD") as much as possible.

Early testing found users sometimes felt uncomfortable without a reference database, especially for combination or prepared foods. Thus, we added a food lookup feature. The on-device database was based on the NutritionistPro Knowledge Base (NPKB), which contains about 42,000 foods. Figure 1d shows results from a database query. The results are split into four tabs: generic (grouped by food class, both as defined in NPKB); brand (grouped by manufacturer); mine (all foods the user has eaten, grouped alphabetically); and recent (grouped by most recently used). Figure 1e shows the details of a specific food. The dark gray blocks indicate the user's daily goal for a component, the light gray blocks indicate how much has been consumed for the day, and the colored boxes indicate how the specified amount will impact the daily totals. The colored blocks also help the user understand the components of the food. The "Add to Journal" button increments the amounts for the relevant components.

The Today screen (Figure 1e) provides a history of entries for the day. An entry is either a single +1 entry, a food from the database, a user-defined combination, or a group of +1 entries. If a user makes many +1 entries in a short period of time (10 seconds), they are grouped together. From this screen, the user can select a row to edit it.

Features not described in this paper include creating custom food entries, editing entries, changing goals, and reviewing weekly activity.

\section{Evaluation}

We performed a two-phase evaluation of POND. The first is an in-lab study where participants created specific food entries. The goal was to collect usage data we could compare across users. In the second phase, the participants used POND in situ for three weeks. This allowed us to understand how POND adapted to real world eating contexts. This paper reports on just the first phase.

This evaluation included 24 participants, 18 female and 6 male. Ages ranged from 21-64 with varying occupations. All participants reported using their mobile phone several times a day, and all but 1 reported entering text on their mobile phones several times a day (the remaining 1 entered text 1-2 times daily). 19 participants reported that one of their health goals was to "Eat better". 17 reported themselves as "very" or "fairly" knowledgeable about food and nutrition, while 5 as "not so" knowledgeable or "fairly uneducated" about food and nutrition.

Participants were recruited via Craigslist, multiple community email lists, and physical posters on community bulletin boards. The recruitment advertisement asked for people who were able and willing to journal their food for three weeks. Participants were compensated $\$ 125$ for participation in both phases of the study. Participants self-reported they had no medical concerns that impacted their food choices and owned their own Android devices.

The study consisted of 4 conditions with 5 tasks in each. The conditions varied in the number of components the user could use to create a food entry. Conditions were Small (2 components), Medium (5 components), Big (9 components), and Full (all 12 components, excluding alcohol). Alcohol was excluded from this study to include participants under the legal drinking age. Components were chosen randomly without replacement to ensure users had equal exposure to all components. Order of conditions for each participant was counterbalanced. Each condition represented roughly 1 day of food intake, and each task represented the content of a single meal. Each food name and amount for a single task was printed on a card. Tasks were presented one at a time. At the end of each condition, participants completed a questionnaire that included TLX workload measures.

\section{RESULTS}

In the interest of space, we focus our discussion on three results. Entry strategy characterizes whether tasks were completed using only the +1 buttons, only the food lookup feature, or a combination. Search terms reveals what foods participants looked up in the database rather than entering directly via the +1 buttons. Likes and dislikes is participant self-report of what they liked and did not like about each condition.

\section{A. Entry strategy}

We were interested in when users made the decision to use the +1 buttons or the lookup feature to enter a task. Participants reflected one of three strategies: overview, detailed and opportunistic. Participants concerned with an overview primarily used the +1 buttons for all tasks. The detailed strategy reflected primarily using the database lookup feature. The opportunistic strategy made frequent use of both +1 entries and database lookup. 10 participants reflected the overview strategy, with 7 participants using the +1 buttons exclusively (i.e., never using the lookup feature). 4 participants reflected the detailed record, primarily using the lookup feature. The remaining 10 participants reflected an opportunistic approach, choosing to make frequent use of both the +1 strategy and the lookup strategy.

\section{B. Search terms}

Overall, the 24 participants made 650 queries from 273 unique phrases. 130 search terms were used by more than one person. There are 13 queries that were made by 8 or more participants. Of these 13 queries, 3 represent foods that most likely fit into a single category (e.g., egg, salad, milk). It is possible the salad query was used to find a "Caesar salad" entry that represent multiple categories (the salad greens plus dressing and croutons), rather than simply salad greens (which could be counted with just one food group). The remaining 10 most common queries are foods that are primarily packaged and prepared (e.g., doritos, wheat thins, fiber one). The mean length of query is 13.6 characters. 6 search terms were between 36 and 40 characters, with as many as 7 words ("bag n season pork chop seasoning mix").

\section{Likes and Dislikes}

Qualitative feedback about the Small condition (2 components) indicated some people liked that it was so short, therefore quick and easy to enter, and "it was trying to just keep track of my best and worst food choices". Others felt it was too limiting: "I wanted to put all of the food I ate into categories, not just some of it".

Feedback about the Medium and Large (5 and 9 components) conditions was mixed. There were fewer components than the Full 
condition, but it was more "mentally taxing" because participants "had to think more about whether a food contained parts of the specified categories". This is possibly due to randomly choosing which components were presented in each condition. The random choices may not reflect meaningful components for the user.

Finally, participants liked having all the categories. They reported it made them more informed and ensured they accounted for all components. Feedback suggested that it was satisfying to have a way to count all foods. When all of the components are present, all food can be counted.

\section{DISCUSSION}

The goal of the in-lab portion of the POND evaluation was to characterize how people used the diary to create known food entries. We reported the strategy participants used to make an entry, the search terms used for known foods, and the things participants liked and disliked.

Participants were divided on how much to use the +1 buttons rather than the lookup feature for creating food entries. The randomization of the components to the conditions could impact the choices that participants made in regards to using the +1 . The Full condition (which contained all components) is comparable across all participants, but in the Small and Medium conditions it is possible that the components contain either just easy food groups (Fruit, Veggies) or all nutrients (Sodium, Sugar). Nutrients are known to be more challenging to count, and people report using the lookup feature for them. A question addressed in the follow-on study is whether participants continued their entry strategy in situ, and whether the strategy changed as participants became more familiar with how to count foods in terms of the components.

Reviewing the most common search results indicates that participants are searching for unfamiliar, processed foods for which it is challenging to identify components. These foods also tend to be higher in sodium and solid fats, which are difficult to estimate without looking up. This is consistent with our initial usage expectations. We expect the in situ study to provide insight as to whether participants learn about the components of different foods and how it impacts their ability to track those foods with the +1 buttons rather than searching the database.

We initially believed that the component-based design of POND could support user customization and prioritization of food components the user wants to track. This could further streamline the entry process, as fewer components take less time to enter. However, the randomly chosen components in this study appeared to confuse participants. A question to address in future work is whether participants find this ability to customize helpful in situ. Another question is whether users actually customize the interface, or simply ignore components in which they are not interested.

\section{CONCLUSION}

The work presented in this paper represents a preliminary evaluation of an index-based nutrition diary. The evaluation suggests that different users have varying desire for nutrition detail. Providing an ability to vary the amount of detail, and therefore the amount of effort required to capture nutrition details, could help users sustain self-monitoring behaviors. Follow-on work not reported in this paper investigated the use of POND for three weeks in the field.

\section{ACKNOWLEDGEMENTS}

This work was supported in part by Nokia Research Palo Alto and by the National Science Foundation under award OAI-1028195.

\section{REFERENCES}

1. Almaghrabi, R., Villalobos, G., Pouladzadeh, P., and Shirmohammadi, S. A Novel Method for Measuring Nutrition Intake Based on Food Image. IEEE Instrumentation and Measurement Technology Conference (I2MTC). 2012. 366-370.

2. American Cancer Society. Global Cancer Facts and Figures. 2011.

3. Andrew, A., Borriello, G., and Fogarty, J. A Usability Comparison of Food Index-Based Nutrition Diaries. Submitted for Review.

4. Burke, L.E., Swigart, V., Turk, M.W., Derro, N., and Ewing, L.J. Experiences of Self-Monitoring: Successes and Struggles During Treatment for Weight Loss. Qualitative Health Research. 19(6). 2009. 815-828.

5. Fanyu Kong and Jindong Tan. DietCam: Regular Shape Food Recognition with a Camera Phone. IEEE International Conference on Body Sensor Networks (BSN). 2011. 127-132.

6. Fengqing Zhu, Bosch, M., Boushey, C.J., and Delp, E.J. An Image Analysis System for Dietary Assessment and Evaluation. IEEE Conference on Image Processing (ICIP). 2010. 1853-1856.

7. Fogg, B.J. Persuasive Technology: Using Computers to Change What We Think and Do. Morgan Kaufman, San Francisco, 2002.

8. Kennedy, E.T., Ohls, J., Carlson, S., and Fleming, K. The Healthy Eating Index: Design and Applications. Journal of the American Dietetic Association 95(10). 1995. 1103-1108.

9. Kimokoti, R.W. and Millen, B.E. Diet, the Global Obesity Epidemic, and Prevention. Journal of the American Dietetic Association. 111(8). 2011. 1137-1140.

10. Lichtenstein, A.H., Appel, L.J., Brands, M., Carnethon, M., Daniels, S., Franch, H.A., Franklin, B., Kris-Etherton, P., Harris, W.S., Howard, B., Karanja, N., Lefevre, M., Rudel, L., Sacks, F., Van Horn, L., Winston, M., and Wylie-Rosett, J. Diet and Lifestyle Recommendations Revision 2006: A Scientific Statement From the American Heart Association Nutrition Committee. Circulation. 114(1). 2006. 82-96.

11. Mamykina, L., Miller, A.D., Grevet, C., Medynskiy, Y., Terry, M.A., Mynatt, E.D., Davidson, P.R. Examining the Impact of Collaborative Tagging on Sensemaking in Nutrition Management. ACM Conference on Human Factors in Computing Systems (CHI). 2011. 657-666.

12. Mamykina, L., Mynatt, E., Davidson, P., and Greenblatt, D. MAHI: Investigation of Social Scaffolding for Reflective Thinking in Diabetes Management. ACM Conference on Human Factors in Computing Systems (CHI). 2008. 477-486.

13. Mattila, E., Parkka, J., Hermersdorf, M., Kaasinen, J., Vainio, J., Samposalo, K., Merilahti, J., Kolari, J., Kulju, M., Lappalainen, R., and Korhonen, I. Mobile Diary for Wellness Management-Results on Usage and Usability in Two User Studies. IEEE Transactions on Information Technology in Biomedicine. 12(4). 2008. 501-512.

14. Noronha, J., Hysen, E., Zhang, H., and Gajos, K.Z. Platemate: Crowdsourcing Nutritional Analysis from Food Photographs. ACM Symposium on User Interface Software and Technology (UIST). 2011. 1-12.

15. Rainie, L. Smartphone Ownership Update: September 2012. 2012.

16. Reddy, S., Parker, A., Hyman, J., Burke, J., Estrin, D., and Hansen, M. Image Browsing, Processing, and Clustering for Participatory Sensing. ACM Workshop on Embedded Networked Sensors (EmNets). 2007, 13-17.

17. Siek, K.A., Connelly, K.H., Rogers, Y., Rohwer, P., Lambert, D., and Welch, J.L. When Do We Eat? An Evaluation of Food Items Input into an Electronic Food Monitoring Application. IEEE Pervasive Health. 2006. 1 -10.

18. Siek, K.A., Connelly, K.H., and Rogers, Y. Pride and Prejudice: Learning How Chronically Ill People Think About Food. ACM Conference on Human Factors in Computing Systems (CHI). 2006. 947-950.

19. Thomas, T. and Pfeiffer, A.F.H. Foods for the Prevention of Diabetes: How Do They Work? Diabetes/Metabolism Research and Reviews. 28(1). 2012. 25-49.

20. Wang, D.-H., Kogashiwa, M., and Kira, S. Development of a New Instrument for Evaluating Individuals' Dietary Intakes. Journal of the American Dietetic Association. 106(10). 2006. 1588-1593. 